\title{
Association of N-Terminal Pro-Brain Natriuretic Peptide, Matrix -Metalloprotinase-9, and 8-Oxo-2 Deoxy Guanosine with the Incidence of Essential Hypertension
}

\author{
Julian Yonan Ismaeil ${ }^{1}$, Shatha Rouf Moustafa ${ }^{2}$, * \\ ${ }^{1}$ Ministry of Health, Aen Kawa, Erbil, Iraq \\ ${ }^{2}$ Clinical Analysis Department, College of Pharmacy, Hawler Medical University, Havalan city, Erbil, Iraq \\ Email address: \\ julian_pharma@yahoo.com (J. Y. Ismaeil), shatha003@yahoo.com (S. R. Moustafa)
}

\section{To cite this article:}

Julian Yonan Ismaeil, Shatha Rouf Moustafa. Association of N-Terminal Pro-Brain Natriuretic Peptide, Matrix -Metalloprotinase-9, and 8Oxo-2 Deoxy Guanosine with the Incidence of Essential Hypertension. American Journal of Internal Medicine.

Vol. 3, No. 2, 2015, pp. 67-77. doi: 10.11648/j.ajim.20150302.14

\begin{abstract}
Background and Objectives: Oxidative stress and inflammation are cooperative events involved in essential hypertension diseases. This study was as a step for elucidating the contribution of $\mathrm{N}$-terminal pro-brain natriuretic peptide, matrix-metalloprotinase-9 and 8-oxo2-deoxyguanosine with the essential hypertension incidence, development, and progression. Aims: The objective was to determine the serum studied parameters levels in essential hypertensive and normotensive subjects, and to assess the association between these biomarkers with the essential hypertension, and to investigate the effect of other confounding factors like stages, age, and gender and finally find the correlation between all studied parameters. Methods: This study was designed to examine the associations between the focused parameters with the essential hypertension on 50 patients of both genders, as well as an equal numbers of matched age-gender healthy adults were also enrolled in this study as a control group. The hypothesis suggesting that oxidative stress and inflammatory processes influence the risk of adverse clinical outcomes are worth investigating. These parameters were analyzed using enzyme linked immunosorbent assay. Statistical analyses were performed with SPSS version 18. Results: Patients with essential hypertension exhibited significantly higher serum focused parameters levels as compared with the control group. The mean serum Nterminal pro-brain natriuretic peptide levels in patients and control groups were $304.33 \pm 204.19,76.52 \pm 20.98 \mathrm{pg} / \mathrm{ml}$ respectively. The mean serum matrix-metalloprotinase- 9 level in patients and control groups were $2.36 \pm 1.1,1.4 \pm 0.8$ respectively. The mean serum 8-oxo-2-deoxy guanosine level in patients and control groups were $170.40 \pm 41.95,84.11 \pm 34.07$ $(\mathrm{mg} / \mathrm{ml})$ respectively. Conclusions: Data suggested an association between these circulating biomarkers with the incidence, development, and progression of essential hypertension. No effect of age and gender present on serum levels of focused biomarkers in both groups. These focused parameters seem to be a simple, non-invasive tools and independent parameters for early detection, assessing disease severity, and involved in the etiology of disease. Data indicated an alteration in the oxidative status and inflammatory processes in patients with essential hypertension. Accordingly, this work was undertaken to assess contribution of the oxidative stress and inflammatory process as a risk factors. In addition, these parameters are regarded as markers with prognostic significance and as potential therapeutic targets. These biomarkers are tools that might aid the physicians in diagnosis and subsequent risk stratification, guiding selection of therapy, secondary prevention, and serving as a target for therapy.
\end{abstract}

Keywords: Essential Hypertension, N-Terminal Pro-Brain Natriuretic Peptide, Matrix-Metalloprotinase-9, 8-Oxo2-Deoxyguanosine

\section{Introduction}

Hypertension is clinically defined as the increased arterial blood pressure (BP) of over $139 \mathrm{~mm} \mathrm{Hg}$ for systolic BP (SBP) and/or $89 \mathrm{~mm} \mathrm{Hg}$ for diastolic BP (DBP). Hypertension is associated with alterations in the cardiac, neural, renal, and vascular control systems, which increase the cardiac output, peripheral resistance, and arterial stiffness. During essential 
hypertension, increased reactive oxygen species (ROS) production leads to enhanced contractility, endothelial dysfunction, inflammation, and increased deposition of extracellular matrix (ECM) proteins. Oxidative stress (OS) and inflammation are cooperative events involved in essential hypertension (EHT) development.

The N-terminal pro-brain natriuretic peptide (NT-proBNP) has emerged as a candidate biomarker that may enable the early detection of essential hypertension.

As a recognized prognostic marker of several cardiac conditions, NT-proBNP has also been proposed to be an indicator for prognosis and follow-up therapy [1, 2]. In addition, the use of NT-proBNP is well established in the diagnosis and staging of patients with EHT. The natriuretic peptides have attracted much interest because they are of diagnostic and prognostic importance.

Hypertension develops as a result of cardiac hypertrophy and fibrosis or as a result of exchange of the ECM [3]. Matrix metalloprotinases (MMPs) are believed to be responsible for the destruction of connective tissue at sites of chronic inflammation. Among the cytokines, tumor necrosis factor- $\alpha(\mathrm{TNF}-\alpha)$ is a pro-inflammatory cytokine that can increase matrix collagen degradation by upregulating MMPs activity and downregulating the tissue inhibitors of matrix metalloprotinases (TIMPs) [4]. The ECM depends on a balance between MMPs and (TIMPs) [5]. Therefore, imbalances between MMPs and TIMPs are involved in the pathological mechanisms of numerous diseases [6]. Oxidative stress results from the overproduction of free radicals (FRs) or the depletion of antioxidants, this type of stress has been implicated in the development of hypertension [7]. The OS by-product (8-oxo-2dG) was previously found to increase in patients with EHT [8].

8-oxo2-deoxyguanosine is an oxidized derivative of deoxyguanosine; this compound is generated by the oxidation of DNA under physiopathological conditions or environmental stress [9]. 8-oxo2-deoxyguanosine has been linked to several biological processes and diseases, including hypertension [8]. Mounting evidence indicates that OS has an important role in the development of EHT. The present study was undertaken to evaluate the OS biomarker 8-oxo-2dG in EHT patients. The quantification of serum 8-oxo-2dG levels is used as a specific marker of oxidative damage to DNA. This parameter has been used to express the oxidation status of EHT patients. Therefore, such parameters appear to be simple, non-invasive tools and independent parameters for the diagnosis and assessment of disease severity. These parameters may contribute to the prognosis of EHT incidence and development, they may also be involved in the etiology of EHT. Accordingly, these selected parameters have emerged as candidate biomarkers that enable the early detection and prognosis of patients with EHT. This study was designed to test the hypothesis that serum concentrations of the selected parameters were altered and related to the incidence, development, and progression of EHT. Moreover, this study sought to evaluate the effect of staging on the concentration of these biomarkers. which were related to
EHT, to determine the effect of other confounding factors, such as the stages of EHT, age, and gender, and to evaluate the correlation between all the studied parameters. Moreover, the study intended to determine the role of the selected parameters as screening biomarkers for the incidence, progression, and development of EHT with the contribution of OS and inflammatory processes as risk factors.

\section{Patients and Methods}

\subsection{Subjects and Study Design}

This prospective study was conducted at the College of Pharmacy of Hawler Medical University in the Kurdistan Region of Iraq from November 2013 to December 2014. A total of 50 patients with EHT of both genders were selected from patients who routinely came to the Internal Medicine Outpatients Clinic at the Hawler Teaching Hospital for follow-up and management. For comparison, an equal number of the matched age-gender healthy adults were also enrolled in this study as a control group. Healthy subjects were selected from the staff and sub-staff of the same hospital. This study was designed to investigate the cooperation of OS concomitant with the inflammatory processes during the prevalence of EHT. Participants were interviewed and informed regarding the nature of the study and verbal consent was obtained from each subject. All procedures were in accordance with established ethical standards. The Ethics Committee of Medical Research at College of Pharmacy of Hawler Medical University approved the study protocol. The control group was confirmed to be normal by biochemical and hematological examinations. This prospective study involved a total of 96 participants. Among these participants, 50 patients of both genders ( 26 men and 24 women) were newly diagnosed with EHT; their mean age at diagnosis was $50.90 \pm 9.27$ years were enrolled in this study after the exclusion of other diseases based on patient clinical history, laboratory investigations, and clinical examination. For purposes of comparison, 46 age-gender matched, apparently healthy adults were randomly selected as the control group, this group included 23 healthy men and 23 healthy women with a mean age of $49.19 \pm 9.49$ years. Both groups completed the baseline questionnaire with selfreported questions concerning several risk factors for EHT, including the history of diabetes, smoking, physical activity, alcohol consumption, hormone replacement therapy, family history, past medical history, and medication history. Case sheets had been prepared for each patient, each including the following information: age, gender, race, occupation, social history, chief complains, other diseases, and medications. This investigation included biochemical and hematological estimates. All clinicopathological data of the patients were collected from the clinical files of the patients. The following exclusion criteria were applied: diabetics; asthmatic patients; malignancies; muscular disease; renal disease; patients with GFR $<30 \mathrm{ml} / \mathrm{min}$, given the reduced renal excretion of NTproBNP at these levels [10], heart failure, arthritis, chronic 
diseases, current use of any medications, which included dietary supplements. Furthermore, patients on lipid-lowering drugs and participants with antioxidant vitamin or mineral supplements were excluded from the current study. Patients with chronic liver disease, endocrine dysfunction, and CHD were also excluded from the study because these diseases also have serum biochemical disturbances. Data depended on the physical and clinical examination as well as laboratory investigations (hematological and biochemical data) to prevent overlapping or interfering results in the present study.

\subsection{Grouping of Patients}

Patients group classified in to stage I, stage II and stage III of essential hypertension according to [11] in order to study the effect of staging on the serum levels of studied parameters.

\subsection{Methods}

\subsubsection{Collection of Sample}

The Protocol of the Study: The fasting blood $(10 \mathrm{ml})$ samples were collected without using tourniquet. The blood samples were left for 30 minutes for coagulation purpose, and then made centrifugation for 15 minutes at 2500-3500 revolution per minutes (rpm).The sera of the participants were separated and divided into several parts and put them into several plastic plain tubes to do the biochemical tests of the current study. The sera of the participants were stored at $\left(-8 \mathrm{C}^{\circ}\right)$ till the day of the analysis. The sera were prepared for measurement by warming the frozen sera at room temperature.

\subsubsection{Biochemical Determinations}

Inflammatory markers NT-proBNP, MMP-9 and oxidative stress marker 8-oxo-2 deoxy guanosine were measured using enzyme linked immunosorbent assay (ELISA).
Data were analyzed by using the Statistical patch for Social Sciences (SPSS vi.18). The results of biochemical tests were expressed as mean \pm standard deviation (SD). Furthermore, student t-test was applied to compare between two means. Multiple regression was used to show the association between each of the biomarkers (as a dependent variables) and several independent variables. A (p) value of $\leq$ 0.05 was considered as statistical significance. Correlations between laboratory findings and continuous variables were evaluated using linear regression analysis.

\section{Results}

\subsection{Subjects Characteristics}

The characteristics of the participants have been shown in (Table 1).This study included 96 participants of both genders, 50 of them were newly diagnosed essential hypertensive patients with mean age of $(50.9 \pm 9.2)$. The reminders 46 were apparently healthy adults with mean age was (49.1 \pm 9.4$)$.

\subsection{Essential Hypertension -Effect}

The variation in the mean serum levels of studied parameters in patient group as compared with the control group has been shown in (Table 1). There were a significant elevation in the serum levels of the studied parameters as compared with the control group $\mathrm{p}<0.001$. Regarding NTproBNP the mean serum level of this parameter was $(304.33 \pm 204.19)(\mathrm{pg} / \mathrm{ml})$ in patients group and it was $(76.52 \pm 20.98)(\mathrm{pg} / \mathrm{ml})$ in control group. The mean serum level of MMP-9 $(2.36 \pm 1.18 \mathrm{SD}) \mathrm{ng} / \mathrm{ml}$ in patient group while the mean serum level of MMP-9 was $(1.42 \pm 0.87) \mathrm{ng} / \mathrm{ml}$ in control group. In case of $80 x 02 \mathrm{dG}$ the mean serum level of it was $(170.40 \pm 41.95)(\mathrm{mg} / \mathrm{ml})$ in patient group and it was $(84.11 \pm 34.07)(\mathrm{mg} / \mathrm{ml})$ in control group.

\subsection{Statistical Analyses}

Table 1. Comparison between patient and control groups regarding the mean serum levels of the studied parameters.

\begin{tabular}{|c|c|c|c|c|c|c|}
\hline Variables & Group & $\mathbf{N}$ & Mean & \pm SD & SE & $\mathbf{P}$ \\
\hline \multirow{2}{*}{ Age } & Patients & 50 & 50.9000 & 9.27857 & 1.31219 & \multirow{2}{*}{0.376} \\
\hline & Control & 46 & 49.1957 & 9.49999 & 1.40070 & \\
\hline \multirow{2}{*}{ Systolic } & Patients & 50 & 16.7000 & 1.61940 & 0.22902 & \multirow{2}{*}{$<0.001$} \\
\hline & Control & 46 & 11.7609 & 0.84155 & 0.12408 & \\
\hline Diastolic & Patients & 50 & 9.6300 & 0.77466 & 0.10955 & $<0.001$ \\
\hline \multirow{2}{*}{ NT-proBNP(pg/ml) } & Patients & 50 & 304.3340 & 204.19890 & 28.87809 & \multirow{2}{*}{$<0.001$} \\
\hline & Control & 46 & 76.5261 & 20.98343 & 3.09384 & \\
\hline \multirow{2}{*}{ MMP-9 (ng/ml) } & Patients & 50 & 2.3638 & 1.18680 & 0.16784 & \multirow{2}{*}{$<0.001$} \\
\hline & Control & 46 & 1.4226 & 0.87738 & 0.12936 & \\
\hline \multirow{2}{*}{8 oxo $2 \mathrm{dG}(\mathrm{mg} / \mathrm{ml})$} & Patients & 50 & 170.4020 & 41.95269 & 5.93301 & \multirow{2}{*}{$<0.001$} \\
\hline & Control & 46 & 84.1196 & 34.07789 & 5.02451 & \\
\hline
\end{tabular}

\subsection{Stage Effect}

The statistical study shows the stage effect on the mean serum levels of the studied parameters. The mean serum levels of focused parameters were increased significantly according to the stage progression of the disease $\mathrm{p}<$ 
0.001(Table 2).

Table 2. The effect of stages of hypertension on serum levels of studied biomarkers.

\begin{tabular}{|c|c|c|c|c|c|}
\hline & & $\mathbf{N}$ & Mean & \pm SD & $\mathbf{p}$ \\
\hline \multirow{4}{*}{ BNP (pg/ml) } & Stage 1 & 14 & 137.679 & 16.186 & \multirow{4}{*}{$<0.001$} \\
\hline & Stage II & 21 & 228.262 & 61.391 & \\
\hline & Stage III & 15 & 566.380 & 174.027 & \\
\hline & Total & 50 & 304.334 & 204.199 & \\
\hline \multirow{4}{*}{ MMP-9 (ng/ml) } & Stage 1 & 14 & 1.164 & 0.223 & \multirow{4}{*}{$<0.001$} \\
\hline & Stage II & 21 & 2.208 & 0.393 & \\
\hline & Stage III & 15 & 3.703 & 1.125 & \\
\hline & Total & 50 & 2.364 & 1.187 & \\
\hline \multirow{4}{*}{$8 \mathrm{OXO}(\mathrm{mg} / \mathrm{ml})$} & Stage 1 & 14 & 124.886 & 38.242 & \multirow{4}{*}{$<0.001$} \\
\hline & Stage II & 21 & 170.776 & 14.085 & \\
\hline & Stage III & 15 & 212.360 & 23.675 & \\
\hline & Total & 50 & 170.402 & 41.953 & \\
\hline
\end{tabular}

\subsection{Age-Factor}

The statistical analysis represented that there were no significant differences between different age categories regarding the mean serum levels of the studied parameters, so there were no age effect on the mean serum levels of focused parameters $p>0.05$ in patient and control groups ( Table 3 and Table 4) respectively .

Table 3. Comparison between different age categories regarding the mean serum levels of the studied parameters in patient group.

\begin{tabular}{|c|c|c|c|c|c|}
\hline 1 & & $\mathbf{N}$ & Mean & \pm SD & $\mathbf{p}$ \\
\hline \multirow{5}{*}{ Systolic } & $<40$ & 7 & 16.71 & 2.29 & \multirow{5}{*}{0.411} \\
\hline & $40-49$ & 16 & 16.69 & 1.49 & \\
\hline & $50-59$ & 17 & 16.29 & 1.36 & \\
\hline & $60+$ & 10 & 17.40 & 1.71 & \\
\hline & Total & 50 & 16.70 & 1.62 & \\
\hline \multirow{5}{*}{ Diastolic } & $<40$ & 7 & 9.86 & 0.80 & \multirow{5}{*}{0.861} \\
\hline & $40-49$ & 16 & 9.59 & 0.88 & \\
\hline & $50-59$ & 17 & 9.56 & 0.61 & \\
\hline & $60+$ & 10 & 9.65 & 0.91 & \\
\hline & Total & 50 & 9.63 & 0.77 & \\
\hline \multirow{5}{*}{ NT-pro BNP(pg/ml) } & $<40$ & 7 & 331.26 & 258.57 & \multirow{5}{*}{0.421} \\
\hline & $40-49$ & 16 & 304.66 & 219.31 & \\
\hline & $50-59$ & 17 & 247.44 & 129.75 & \\
\hline & $60+$ & 10 & 381.69 & 242.95 & \\
\hline & Total & 50 & 304.33 & 204.20 & \\
\hline \multirow{5}{*}{ MMP-9 (ng/ml) } & $<40$ & 7 & 2.31 & 1.43 & \multirow{5}{*}{0.124} \\
\hline & $40-49$ & 16 & 2.33 & 0.89 & \\
\hline & $50-59$ & 17 & 1.98 & 0.82 & \\
\hline & $60+$ & 10 & 3.11 & 1.70 & \\
\hline & Total & 50 & 2.36 & 1.19 & \\
\hline \multirow{5}{*}{8 oxo dG $(\mathrm{mg} / \mathrm{ml})$} & $<40$ & 7 & 167.16 & 70.51 & \multirow{5}{*}{0.572} \\
\hline & $40-49$ & 16 & 173.69 & 25.57 & \\
\hline & $50-59$ & 17 & 160.75 & 30.18 & \\
\hline & $60+$ & 10 & 183.83 & 56.17 & \\
\hline & Total & 50 & 170.40 & 41.95 & \\
\hline
\end{tabular}

Table 4. Comparison between different age categories regarding the mean serum levels of the studied parameters in control group.

\begin{tabular}{|c|c|c|c|c|c|}
\hline & & $\mathbf{N}$ & Mean & \pm SD & $\mathbf{P}$ \\
\hline \multirow{5}{*}{ systolic } & $<40$ & 7 & 11.43 & 0.53 & \multirow{5}{*}{0.252} \\
\hline & $40-49$ & 16 & 11.56 & 0.73 & \\
\hline & $50-59$ & 16 & 12.06 & 0.83 & \\
\hline & $60+$ & 7 & 11.86 & 1.21 & \\
\hline & Total & 46 & 11.76 & 0.84 & \\
\hline \multirow{5}{*}{ diastolic } & $<40$ & 7 & 7.14 & 0.75 & \multirow{5}{*}{0.383} \\
\hline & $40-49$ & 16 & 7.66 & 0.60 & \\
\hline & $50-59$ & 16 & 7.59 & 0.74 & \\
\hline & $60+$ & 7 & 7.64 & 0.63 & \\
\hline & Total & 46 & 7.55 & 0.68 & \\
\hline \multirow{5}{*}{ NT-proBNP (pg/ml) } & $<40$ & 7 & 82.59 & 24.61 & \multirow{5}{*}{0.628} \\
\hline & $40-49$ & 16 & 71.68 & 24.01 & \\
\hline & $50-59$ & 16 & 79.49 & 18.21 & \\
\hline & $60+$ & 7 & 74.77 & 17.13 & \\
\hline & Total & 46 & 76.53 & 20.98 & \\
\hline \multirow{5}{*}{ MMP-9 (ng/ml) } & $<40$ & 7 & 1.25 & 0.67 & \multirow{5}{*}{0.816} \\
\hline & $40-49$ & 16 & 1.55 & 1.01 & \\
\hline & $50-59$ & 16 & 1.32 & 0.71 & \\
\hline & $60+$ & 7 & 1.54 & 1.17 & \\
\hline & Total & 46 & 1.42 & 0.88 & \\
\hline \multirow{5}{*}{8 oxodG(mg/ml) } & $<40$ & 7 & 58.50 & 22.86 & \multirow{5}{*}{0.105} \\
\hline & $40-49$ & 16 & 94.21 & 34.69 & \\
\hline & $50-59$ & 16 & 88.79 & 35.43 & \\
\hline & $60+$ & 7 & 76.00 & 29.48 & \\
\hline & Total & 46 & 84.12 & 34.08 & \\
\hline
\end{tabular}

\subsection{Gender-Effect}

Statistical study revealed that there were no significant 
differences between men and women regarding mean serum levels of the studied parameters in patient and control groups

effect.

(Table 5 and Table 6 ) respectively, so there were no gender

Table 5. Comparison between men and women regarding the serum levels of the studied parameters in patients group.

\begin{tabular}{|c|c|c|c|c|c|c|}
\hline & Gender & $\mathbf{N}$ & Mean & \pm SD & SE & p \\
\hline \multirow{2}{*}{ Age } & Male & 26 & 51.654 & 10.024 & 1.966 & \multirow{2}{*}{0.555} \\
\hline & Female & 24 & 50.083 & 8.536 & 1.742 & \\
\hline \multirow{2}{*}{ Systolic } & Male & 26 & 16.885 & 1.657 & 0.325 & \multirow{2}{*}{0.407} \\
\hline & Female & 24 & 16.500 & 1.588 & 0.324 & \\
\hline \multirow{2}{*}{ Diastolic } & Male & 26 & 9.654 & 0.704 & 0.138 & \multirow{2}{*}{0.823} \\
\hline & Female & 24 & 9.604 & 0.859 & 0.175 & \\
\hline \multirow{2}{*}{ NT-proBNP (pg/ml) } & Male & 26 & 317.235 & 209.041 & 40.996 & \multirow{2}{*}{0.647} \\
\hline & Female & 24 & 290.358 & 202.345 & 41.303 & \\
\hline \multirow{2}{*}{ MMP-9 (ng/ml) } & Male & 26 & 2.539 & 1.393 & 0.273 & \multirow{2}{*}{0.282} \\
\hline & Female & 24 & 2.174 & 0.906 & 0.185 & \\
\hline \multirow{2}{*}{ 8-oxo-2dG (mg/ml) } & Male & 26 & 173.485 & 43.557 & 8.542 & \multirow{2}{*}{0.594} \\
\hline & Female & 24 & 167.063 & 40.805 & 8.329 & \\
\hline
\end{tabular}

Table 6. Comparison between men and women regarding the serum levels of the studied parameters in control group.

\begin{tabular}{|c|c|c|c|c|c|c|}
\hline & Gender & $\mathbf{N}$ & Mean & \pm SD & SE & $\mathbf{p}$ \\
\hline \multirow{2}{*}{ Age } & Male & 23 & 47.08 & 9.59 & 2.00 & \multirow{2}{*}{0.13} \\
\hline & Female & 23 & 51.30 & 9.12 & 1.90 & \\
\hline \multirow{2}{*}{ Systolic } & Male & 23 & 11.97 & 0.57 & 0.12 & \multirow{2}{*}{0.08} \\
\hline & Female & 23 & 11.54 & 1.01 & 0.21 & \\
\hline \multirow{2}{*}{ Diastolic } & Male & 23 & 7.56 & 0.62 & 0.13 & \multirow{2}{*}{0.91} \\
\hline & Female & 23 & 7.54 & 0.73 & 0.15 & \\
\hline NT-proBNP (pg/ml) & Male & 23 & 80.74 & 20.51 & 4.27 & 0.175 \\
\hline \multirow{2}{*}{ MMP-9 (ng/ml) } & Male & 23 & 1.34 & 0.82 & 0.17 & \multirow{2}{*}{0.536} \\
\hline & Female & 23 & 1.50 & 0.93 & 0.19 & \\
\hline \multirow{2}{*}{ 8-oxo-2dG (mg/ml) } & Male & 23 & 77.65 & 23.98 & 5.00 & \multirow{2}{*}{0.201} \\
\hline & Female & 23 & 90.58 & 41.38 & 8.62 & \\
\hline
\end{tabular}

\subsection{The Correlation Coefficient}

Table 7. The correlation coefficient between the studied parameters in patients group.

\begin{tabular}{lllll}
\hline X variable & Y variable & R & P value & N \\
\hline Age & MMP-9 & 0.1 & 0.2 & 50 \\
Age & NT-pro BNP & 0.05 & 0.6 & 50 \\
Age & 8-oxodG & 0.05 & 0.7 & 50 \\
SBP & MMP-9 & 0.9 & $<0.001$ & 50 \\
SBP & NT-proBNP & 0.9 & $<0.001$ & 50 \\
SBP & 8-oxodG & 0.8 & $<0.001$ & 50 \\
DBP & MMP-9 & 0.4 & 0.001 & 50 \\
DBP & NT-pro BNP & 0.3 & 0.005 & 50 \\
DBP & 8-oxodG & 0.4 & 0.003 & 50 \\
MMP-9 & NT-pro BNP & 0.9 & $<0.001$ & 50 \\
MMP-9 & 8-oxodG & 0.8 & $<0.001$ & 50 \\
NT-pro BNP & 8-oxodG & 0.7 & $<0.001$ & 50 \\
\hline
\end{tabular}

The statistical study investigate the correlation coefficient between the studied parameters in patient group, there were non significant weak correlation between age and MMP-9, non significant weak correlation between age and NT-pro BNP,non significant weak correlation between age and 8oxo-2dG,significant strong correlation between SBP and MMP-9, significant strong correlation between SBP and NTpro BNP,significant strong correlation between SBP and 8oxo-2dG , significant moderate correlation between DBP and MMP-9, significant weak correlation between DBP and NTpro BNP, significant moderate correlation between DBP and
8-oxodG, Significant strong correlation between MMP-9 and NT-pro BNP, significant strong correlation between MMP-9 \& 8-oxodG, significant strong correlation between NT-pro BNP and 8-oxodG (Table 7).

\section{Discussion}

\subsection{General View}

The rational of the present study conducted the association oxidative stress / antioxidant status imbalance concomitant with inflammatory process in patients with EHT. This study has highlighted three markers that are considered integral to the relationship between oxidant /antioxidant status imbalance and inflammatory process with the incidence, development, and progression of EHT, these markers are NTproBPN, MMP-9, and 8-oxo- 2deoxyguanosine.

All of these parameters have been correlated with the most important clinical indices of patients such as stage of disease, age and gender.

\subsection{Effect of Essential Hypertension on the Serum Levels of Studied Biomarkers}

\subsubsection{Inflammatory Biomarker N-Terminal Pro-Brain Natriuretic Peptide}

This study revealed a significant elevation in the serum levels of NT-proBNP as compared with the control group $\mathrm{p}<$ 
0.001 (Table 1). So, this finding could indicate that there was a significant relationship between serum NT-proBNP level as an inflammatory marker with incidence of EHT. Accordingly, elevated serum NT-proBNP levels have been shown to be a predictive and contributor marker for the prevalence of EHT. It was hypothesized that a significant elevation in serum NTproBNP level could indicate it's association with EHT. The present data supported the hypothesis that the NT-proBNP serum levels may play a key role in the inflammatory response that contributed in EHT incidence.

An increase in NT-proBNP serum concentrations was hypothesized to indicate its association with EHT and, could be associated with the inflammatory status. A link between NT-proBNP and the inflammatory activation process was previously estimated. The present data supported the hypothesis that NT-proBNP serum levels may have a key role in the inflammatory response that contributed in EHT incidence. Taken together, the above mentioned finding confirmed the notion that the inflammatory process is involved with the prevalence of EHT. Accordingly, this finding may account for the correlation of NT-proBNP levels with the etiology of EHT and could be a marker that can be implicated in the pathogenesis of EHT.

This finding addresses the prognostic value of NT-proBNP in EHT, which was confirmed by previous studies [12-15], where in plasma NT-proBNP levels had a significant prognostic value in hypertensive patients. The current result was consistent with the previous findings [12, 14-26]. The significant elevation of NT-proBNP in EHT could be explained by the expression and secretion of NT-proBNP from ventricular myocytes, which is a landmark of cardiac remodeling that is of particular interest in hypertension [25]. $\mathrm{N}$-terminal pro-brain natriuretic peptide is mainly secreted by the left cardiac ventricle as a consequence of pressure overload and wall stretching [27]. Natriuretic peptides are related to systolic dysfunction [28] and increased the filling pressure [29]. This peptide also strongly depends on ventricular after-load, particularly the BP [22] and aortic stiffness. This study showed that serum NT-proBNP was an emerging powerful circulating marker and prognostic noninvasive indicator that could be associated with the EHT. N-terminal pro-brain natriuretic peptide may act as an early predictor of EHT and may be considered a potential candidate marker for diagnosis and follow-up. Therefore, NT-proBNP can be used as a useful biomarker to provide diagnostic information concerning the development and management of EHT as well as a strong prognostic marker associated with the prevalence of EHT. Moreover, NTproBNP acts as an integrative marker that can predict outcomes beyond cardiac events, this peptide may be a useful tool for monitoring the follow-up of patients with EHT. Therefore, NT-proBNP was added as independent prognostic information and could be used to monitor essential hypertensive patients.

4.2.2. Inflammatory Biomarker Matrix Metalloproteinase-9 Given that EHT may be a part of an inflammatory disease, the circulatory factors related to inflammation could be predictors of EHT prevalence. Nevertheless, increasing evidence supports the relationship between the elevation of vascular inflammatory markers and hypertension [30]. Matrixmetalloproteinase-9 was hypothesized to play an independent role in BP modulation in patients with EHT. This study was aimed to elucidate whether serum MMP-9 levels were related to EHT. Results demonstrated that the significant elevation in the serum MMP-9 levels in the patient group as compared with the control group $\mathrm{p}<0.001$ (Table 1). Accordingly, the inflammatory marker MMP-9 is considered a contributory marker of BP in EHT. This finding provides an important link between the risk factors of inflammation and the prevalence of EHT. Therefore, inflammation is implicated in the pathogenesis of EHT.

In addition, it was supported the current study and stated that the elevation of plasma MMP-9 has been associated with increased arterial stiffness and elevated BP in EHT [31]. The elevation of MMP-9 is important because abnormal MMP levels can stimulate vascular inflammation, which is a potential contributor to the pathogenesis and progression of EHT [32,33]. The elevation of MMP-9 predicts worse outcomes in patients with EHT. The current result was consistent with the findings of several previous studies [31, 33-37]. Therefore, the significant elevation of serum MMP-9 levels was associated with the incidence of EHT and may be explained by the well-known contribution of inflammation to the incidence of EHT and target organ damage. The MMP-9 enzyme is an inflammatory mediator that may contribute to EHT and its target organ consequences. Matrixmetalloproteinases were previously implicated in the development of chronic vascular diseases, but the key effectors and mechanism of action remain unknown [38]. By contrast, they were suggested that plasma MMP-9 is actually decreased in hypertensive subjects as compared to the controls [39,40]. Another study reported no significant differences in the MMP-9 levels between hypertensive patients and controls [41].

The present prospective study gave available evidence to support the link between inflammation and EHT prevalence as well as to discuss future implications for the drug therapy of EHT.

\subsubsection{Oxidative Stress Marker 8-Oxo-2 Deoxy Guanosine}

The present study was designed to simultaneously assess the OS levels by measuring 8-oxo- $2 \mathrm{dG}$ in the patient and control groups. The study aimed to test the OS as a potential early risk factor for EHT prevalence in the Hawler population by examining 8-oxo-2dG as a byproduct of ROS-induced DNA damage. The statistical analysis revealed that the OS marker 8-oxo-2dG was significantly increased in the patient group as compared with the control group $\mathrm{p}<0.001$ (Table 1 ). The result of the current study was concordant with several previous findings [42-45]. The present study was designed to assess the serum 8-oxo-2dG levels to express the degree of OS in patients with EHT. Therefore, the hypothesis that OS serves as a risk factor of EHT incidence was tested. Results 
strongly suggested that the serum 8-oxo-2dG level can serve as a sensitive biomarker of oxidative DNA damage and OS is important in the pathogenesis of EHT. The results confirmed the hypothesis that EHT involves a state of OS, which is implicated and contributes to the pathogenesis of EHT.

This study clearly demonstrated a significant association between the serum 8-oxo-2dG levels and the incidence of EHT and confirmed the idea that OS has an important role in the incidence of EHT. Accordingly, the 8-oxo-2dG assay is a good marker for monitoring OS changes in patients with EHT. A previous study on hypertensive patients reported that the amount of damaged 8 -oxo- $2 \mathrm{dG}$ in hypertensive patients was significantly higher than that of the control subjects [42]. Meanwhile, urinary sample from hypertensive patients was analysed and reported that urinary 8 -oxo- $2 \mathrm{dG}$ was a reliable OS marker in hypertension and 8-oxo-2dG levels in hypertensive patients was significantly higher than those of the controls [43]. Moreover, urinary samples of EHT patients was investigated and reported that 8 -oxo-2dG was significantly higher in EHT patients than in the control group [46].

Several lines of evidence show that oxidative damage may be an important mechanism underlying several pathophysiological states such as EHT. The present study provided evidence that oxidative DNA damage was associated with the incidence of EHT. According to the present study, OS has been implicated in the pathogenesis of EHT. Mounting evidence indicates that OS has an important role in the incidence of EHT. Therefore, this study has offered the opportunity to test whether OS could be a potential early risk factor for developing EHT.

\subsection{Stage-Effects}

\subsubsection{NT-ProBNP}

In the patient group, the serum NT-proBNP level was significantly increased according to the stage progression $\mathrm{p}<$ 0.001 (Table 2). Therefore, according to the current result, the elevated NT-proBNP level serves as a simple, noninvasive tool and independent parameter for assessing disease severity in patients with EHT and it might be related with development and progression of EHT. The analysis of this biomarker was based upon an evaluation of the utility of serum NT-proBNP to indicate the incidence, development, and progression of EHT. Unlike the present study, it was reported that no differences were demonstrated between moderate and severe stages in patients with EHT in a small study [47]. Recent advances in basic science have established a fundamental role for inflammation in mediating all stages of this disease from initiation until the progression of EHT.

\subsubsection{Matrixmetalloprotenase -9}

In the patient group, the serum MMP-9 level was significantly increased according to the stage progression $\mathrm{p}<$ 0.001(Table 2). Therefore, this noninvasive marker could indicate the severity of EHT and it might be related with development and progression of EHT. Accordingly, current data supported the hypothesis that elevated MMP-9 levels were associated with the progression and development of EHT. Similarly, it was reported that MMP-9 has been implicated in the development and progression of EHT[30].

\subsubsection{8-Oxo2deoxy Guanosine}

In the patients group, the serum 8-oxo-2dG level was increased significantly according to the stage progression $\mathrm{p}<$ 0.001 (Table 2). The current study indicated that free radicalmediated oxidative damage of DNA occurs at an early stage of EHT and could increase significantly in later stages. Therefore, the present study was designed to assess the serum 8-oxo-2dG levels to express the degree of OS in essential hypertensive patients and to clarify the severity of disease by investigating the staging of EHT. Therefore, this noninvasive marker could indicate the severity of EHT and was associated with progression and development of EHT.

\subsection{Age-Factor}

This study attempted to determine the age effect on the serum levels of studied parameters. The statistical analysis found no significant differences between the different age categories in terms of the serum levels of the studied parameters. Therefore, no age effects $p>0.05$ were found in the patient and control groups (Table 3 and Table 4, respectively). In addition (Table 7) has shown that there were weak non significant correlation between the age and studied parameters. Unlike the current findings, it was reported that NT-proBNP was significantly associated with age and DBP [48]. Meanwhile, previous studied on MMP-9 were carefully reviewed, there were no any results that deal with age effect on the serum level of MMP-9,accordingly this study might be the first one that deal with association of age factor with MMP-9 in patients with EHT. In addition, it was reported that damaged 8-oxo-2dG was found in elderly people [49].

\subsection{Gender-Factor}

This study also highlighted one of the aspects regarding gender effect. No significant differences were observed between men and women in terms of the serum levels of the studied parameters in patients and control groups (Tables 5 and 6 , respectively). The patient group of the current study consisted of 26 men with a mean age of $51.654 \pm 10.02$ years and 24 women with a mean age of $50.083 \pm 8.536$ years. Meanwhile, the control group included 23 men with a mean age of $47.087 \pm 9.596$ years and 23 women with a mean age of $51.304 \pm 9.123$ years (Table 1 ). The absence of gender effects could be attributed to the fact that women who enrolled in this study were elderly (post-menopause). Thus, no hormonal effects were present. At a young age, the prevalence of hypertension is reportedly higher among men compared to women [50]. This trend can be attributed to the fact that women are more protected from OS by estrogen [51] Contrary to the current findings, it was reported that plasma NT-proBNP levels increase with age and are higher in women than men after the adjustment for age [52]. In addition, it was reported that NT-proBNP was significantly higher $\mathrm{P}<0.001$ in women than in men [24]. It was also 
published that NT-proBNP levels in a healthy population are dependent on age and gender[53].

\subsection{Correlation Coefficient}

The statistical study reveals that there were a significant strong correlation between SBP and studied parameters NTproBNP $\mathrm{r}=0.9 \mathrm{p}<0.001$,MMP-9 $\mathrm{r}=0.9 \mathrm{p}<0.001$, and 8oxo2dg $\mathrm{r}=0.8 \mathrm{p}<0.001$ (Table 7), these strong significant correlations were in harmony with the hypothesis that these parameters NT-proBNP,MMP-9 (parameters of inflammation) and 8-oxo2dg (parameter of oxidative damage of DNA) are associated with EHT incidence and the contribution of the inflammation and OS with the prevalence of EHT. In addition there were a weak significant correlation between DBP and studied parameters as well as there were a strong significant correlation between all studied parameters (Table7). To the best of our knowledge, this is the first report that used Iraq/Kurdistan Governorate population data to evaluate the association between serum levels of selected parameters and the incidence, development, and progression of EHT. Whether inflammation is a cause or effect of EHT remains unclear, but evidence from human and animal studies suggests that inflammation can lead to the development of hypertension. OS and endothelial dysfunction are known to be associated with inflammation and can contribute to hypertension by exacerbating the inflammatory response

The strengths of this study include the use of an equal number of participants from both genders. In addition, this study focused on the association between NT-pro-BNP, MMP-9, and the OS marker 8-oxo-2dG, which have not been fully investigated in the Iraqi Kurdistan Region. Therefore, this study offers the opportunity to test whether the inflammatory status and OS could be a potential early risk factor for the incidence of EHT.

Clinical Implications: Cardiac biomarkers are tools that should aid the physician in diagnosis and/or the subsequent risk stratification for secondary prevention. In some cases, these biomarkers serve as targets for therapy and, finally, for guiding the selection of therapy. The identification of individuals at high risk for cardiovascular events is important for the optimal use of primary and secondary prevention measures. The selected parameters in this study are simple and noninvasive. Thus, the repeated measurement of these parameters may be helpful as part of the evaluation of treatment in patients with EHT and, in particular, as a guide to the selection and timing for alternative therapies. The selected parameters have also been proposed as indicators for follow-up therapy and prognosis. This study supports the expanding evidence that the selected parameters may serve as surrogate markers in the clinical evaluation and management of EHT. Accordingly, these parameters are regarded as markers with prognostic significance and as potential therapeutic targets. Therefore, efforts have been focused on identifying the risk factors for developing EHT, and these parameters may allow for the early detection of people who are likely to develop EHT and the subsequent risk of cardiovascular events. Consequently, prevention and treatment may be initiated earlier.

\section{Conclusion}

Available data revealed the significant elevation in serum levels of selected parameters in association with EHT. Results suggested a strong association between the serum levels of selected parameters with EHT. The serum levels of the studied parameters were significantly elevated according to the stage progression of the EHT. These results indicated that the selected biomarkers are associated with disease progression and development. Therefore, these biomarkers can be considered as independent parameters for assessing disease severity in patients with EHT. These parameters could provide a relatively simple, non-invasive method for investigating EHT. The studied parameters are sensitive biochemical markers, which are valuable and useful tools to diagnose patients with EHT. These markers have also been proposed as indicators for the incidence, development, and progression of EHT, as well as its follow-up and prognosis. Oxidative stress and inflammatory processes may serve as risk factors for the incidence of EHT in the Hawler population. Mounting and cumulative evidence support and indicate that OS and inflammatory processes have an important role in the development and pathogenesis of EHT. The results of the current study supported the role of chronic low-grade inflammation in the incidence of EHT. Data suggested that inflammation may have an integral part in the development and progression of EHT. The significant elevation of the inflammatory parameters NT-proBNP and MMP-9 in patients with EHT are particularly relevant and creates options for potential primary prevention strategies. In addition, the findings of the current study indicated and supported the idea that OS is a cooperative event involved in the incidence, development, and progression of EHT, based on the investigation of 8 -oxo- $2 \mathrm{dG}$ as an oxidative marker of DNA damage. Based on these results, the studied parameters can be regarded as valuable markers associated with the incidence of EHT; these markers should be used as simple, diagnostic, and strong prognostic tools for EHT incidence and risk stratification.

Further prospective studies are needed to evaluate whether lowering of the oxidative stress and inflammatory process, such as via antioxidant treatment and anti-inflammatory therapy, subsequently decreases the incidence, development, and progression of EHT. Efforts have been focused on identifying risk factors for developing EHT; these studies may allow for the early detection of people who are likely to develop EHT and the subsequent risks of cardiovascular events. Thus, the prevention and treatment may be initiated earlier.

\section{Abbreviations}

8-oxo 2dG: 8-oxo2-deoxy guanosine, BP: blood pressure, DBP: diastolic blood pressure, EHT: Essential hypertension, 
MMP-9: Matrix metalloproteinase -9, NT-proBNP: Nterminal Pro-Brain Natriuretic Peptide, OS: oxidative stress, Ref: Reference, ROS: reactive oxygen species, SBP: systolic blood pressure

\section{References}

[1] Olsson LG, Swedberg K, Cleland JG, Spark PA, Komajda M, Metra M, Torp-Pedersen C, Remme WJ, Scherhag A, PooleWilson P. (2007). Prognostic importance of plasma NT-pro BNP in chronic heart failure in patients treated with a betablocker: results from the Carvedilol Or Metoprolol European Trial (COMET) trial. Eur J Heart Fail, 9 :795- 801.

[2] Lainchbury JG, Troughton RW, Strangman KM, Frampton CM, Pilbrow A, Yandle TG, Hamid AK, Nicholls MG, Richards AM. (2010). N-terminal pro-B-type natriuretic peptide-guided treatment for chronic heart failure: results from the battlescarred (NT-proBNP-Assisted Treatment To Lessen Serial Cardiac Readmissions and Death) trial. J Am Coll Cardiol, 55:53-60.

[3] Lee YH, Kim TY, Hong YM. (2009). Metalloproteinase-3 genotype as a predictor of cardiovascular risk in hypertensive adolescents. Korean circulation journal, 39 (8): 328- 334.

[4] Murray DB, Levick SP, Brower GL, Janicki JS. (2010). Inhibition of matrix metal-loproteinase activity prevents increases in myocardial tumor necrosis factor-alpha. J Mol Cell Cardiol, 49: 245-250.

[5] Benjamin MM, Khalil RA. (2012). Matrix metalloproteinase inhibitors as investigative tools in the pathogenesis and management of vascular disease. EXS, 103:209-279.

[6] Fingleton B. (2007). Matrix metalloproteinases as valid clinical targets. Curr pharm Des, 13 (3): 333- 346.

[7] Ogita H, Liao J. (2004). Endothelial function and oxidative stress. Endothelium, 11: 123- 132.

[8] Delaney S, Jarem DA, Volle CB, Yennie CJ. (2012). Chemical and biological consequences of oxidatively damaged guanine in DNA. Free Radic Res, 46 (4): 420- 441.

[9] Patra A, Nagy LD, Zhang Q, Su Y, Müller L, Guengerich FP, Egli M. (2014). Kinetics, Structure, and Mechanism of 8-Oxo7,8-dihydro-2'-deoxyguanosine Bypass by Human DNA Polymerase. J Biol Chem.2014; doi: 10.1074/jbc.M114.551820.

[10] Vivek Thakkar' Wendy M Stevens' David Prior' Owen A Moore Jillian Byron' Danny Liew, Karen Patterson' Pravin Hissaria Janet Roddy' Jane Zochling' Joanne Sahhar, Peter Nash Kathleen Tymms' David Celermajer' Eli Gabbay' Peter Youssef' Susanna M Proudman and Mandana Nikpour, (2012). Nterminal pro-brain natriuretic peptide in a novel screening algorithm for pulmonary arterial hypertension in systemic sclerosis: a case-control study. Arthritis Research \& Therapy, 114: 143.

[11] Joint National Committee on Prevention, Detection, Evaluation, and Treatment of High Blood Pressure, (1997). The Sixth Report of the Joint National Committee on Prevention, Detection, Evaluation, and Treatment of High Blood Pressure (JNC VI). Arch Intern Med, 157 :2413- 2446.

[12] Olsen MH, Wachtell K, Tuxen C, Fossum E, Bang LE, Hall C,
Ibsen H,Rokkedal J, Devereux RB, Hildebrandt P. (2004). Nterminal pro-brain natriuretic peptide predicts cardiovascular events in patients with hypertension and left ventricular hypertrophy: a LIFE study. J Hypertens, 22: 1597- 1600.

[13] Pedersen F, Raymond I, Kistorp C, Sandgaard N, Jacobsen P, Hildebrandt P. (2005). N-terminal pro-brain natriuretic peptide in arterial hypertension: a valuable prognostic marker of cardiovascular events. J Card Fail, 11: 70 - 75.

[14] Olsen MH, Wachtell K, Nielsen OW, Hall C, Wergeland R, Ibsen H, Kjeldsen SE, Devereux RB, Dahlof B, Hildebrandt PR. (2006). N-terminal brain natriuretic peptide predicted cardiovascular events stronger than highsensitivity C-reactive protein in hypertension: a LIFE substudy. J Hypertens, 24:1531- 1539 .

[15] Vinciane Paget, Liliana Legedz, Nathalie Gaudebout, Nicolas Girerd, Giampiero Bricca, Hugues Milon,Madeleine Vincent, Pierre Lantelme, (2011). N-Terminal Pro-Brain Natriuretic Peptide A Powerful Predictor of Mortality in Hypertension. Hypertension, 57: 702- 709.

[16] Per Hildebrandt, Mikael Boesen, Michael Olsen, Kristian Wachtell and Bjoern Groenning, (2004). N-terminal pro brain natriuretic peptide in arterial hypertension-a marker for left ventricular dimensions and prognosis. Eur J Heart Fail, 6 (3): $313-317$.

[17] Kenan İltümür, Aziz Karabulut, Nizamettin Topreak, (2005). NT-proBNP in Isolated Diastolic Dysfunction. Dicle Tip Dergisi, 32 (4): 165- 171.

[18] Mueller T, Gegenhuber A, Dieplinger B, Poelz W, Haltmayer M.(2005) . Capacity of B-type natriuretic peptide (BNP) and amino-terminal proBNP as indicators of cardiac structural disease in asymptomatic patients with systemic arterial hypertension. Clin Chem, 51: 2245 - 2251.

[19] Halse KG, Lindegaard MLS, Goetze JP, Damm P, Mathiesen ER, Nielsen LB. (2005). Increased pro-B-type natriuretic peptide in infants of women with type I diabetes. Clin Chem, 51: 2296- 2302 .

[20] Conen D, Zeller A, Pfisterer M, Martina B. (2006). Usefulness of B-type natriuretic peptide and $\mathrm{C}$-reactive protein in predicting the presence or absence of left ventricular hypertrophy in patients with systemic hypertension. Am J Cardiol, 97: $249-252$.

[21] Belluardo P, Cataliotti A, Bonaiuto L, Giuffre E, Maugeri E, Noto P,Orlando G, Raspa G, Piazza B, Babuin L, Chen HH, Martin FL, McKie PM, Heublein DM, Burnett JC Jr, Malatino LS. (2006). Lack of activation of molecular forms of the BNP system in human grade 1 hypertension and relationship to cardiac hypertrophy. Am J Physiol Heart Circ Physiol, 291: $1529-1535$.

[22] Mouly-Bertin C, Bissery A, Milon H, Dzudie A, Rabilloud M, Bricca G,Vincent M, Lantelme P. (2008). N-terminal pro-brain natriuretic peptide: a promising biomarker for the diagnosis of left ventricular hypertrophy in hypertensive women. Arch Cardiovasc Dis, 101:307-315.

[23] Morillas P, Castillo J, Quiles J, Nunez D, Guillen S, Maceira A, Rivera M, Bertomeu V. (2008). Usefulness of NT-proBNP level for diagnosing leftventricular hypertrophy in hypertensive patients: a cardiac magnetic resonance study. Rev Esp Cardiol, 61:972- 975. 
[24] Toda K' Sato Y, Hara T, Hijiya K, Kaneko R, Okada T, Takatsu Y, Fujiwara H, Iwasaki T. (2010). Correlates of NT-proBNP concentration in patients with essential hypertension in absence of congestive heart failure. J Clin Lab Anal, 24 (1):12- 6

[25] Giampiero Bricca, Pierre Lantelme, (2011). Natriuretic peptides: Ready for prime-time in hypertension? Elsevier, 104 (6-7): 403-409.

[26] Paget V, Legedz L, Gaudebout N, Girerd N, Bricca G, Milon H. (2011). N-terminal pro-brain natriuretic peptide. A powerful predictor of mortality in hypertension. Hypertension, 57:702- 709 .

[27] Alexis E. Malavazos1, Lelio Morricone1, Alessandro Marocchi, Federica Ermeticil, Bruno Ambrosi and Massimiliano M. Corsi, (2006). N-Terminal Pro-B-Type Natriuretic Peptide and Echocardiographic Abnormalities in Severely Obese Patients: Correlation with Visceral Fat .Clinical Chemistry, 52 (6): 1211- 1213.

[28] Mueller T, Gegenhuber A, Poelz W, Haltmayer M. (2004). Head-to-head comparison of the diagnostic utility of BNP and NT-proBNP in symptomatic and asymptomatic structural heart disease. Clin Chim Acta, 341: 41- 48.

[29] Tschope C, Kasner M, Westermann D. (2005). The role of NTproBNP in the diagnostics of isolated diastolic dysfunction: correlation with echocardiographic and invasive measurements. Eur Heart J, 26:2277.

[30] Bautista LE. (2003). Inflammation, endothelial dysfunction, and the risk of high blood pressure: epidemiologic and biological evidence. J HumHypertens, 17: 223- 230.

[31] Zhou S, Feely J, Spiers JP, Mahmud A. (2007). Matrix metalloproteinase-9 polymorphism contributes to blood pressure and arterial stiffness in essential hypertension. J Hum Hypertens, 7 (21): 861- 867.

[32] Boos CJ, Lip GY. (2006). Is hypertension an inflammatory process? Curr Pharm Des, 12:1623-1635.

[33] Raffetto JD, Khalil RA. (2008). Matrix metalloproteinases and their inhibitors in vascular remodeling and vascular disease. Biochem Pharmacol, 75:346 - 359.

[34] Derosa G, D'Angelo A, Ciccarelli L. (2006). Matrix Metalloproteinase-2, -9, and Tissue Inhibitor of Metalloproteinase-1 in Patients with Hypertension. Endothelium, 13: 227-231.

[35] Tan J, Hua Q, Xing X,Wen J, Liu R, Yang Z. (2007). Impact of the metalloproteinase-9/tissue inhibitor of metalloproteinase-1 system on large arterial stiffness in patients with essential hypertension. Hypertens Res, 30: 959963.

[36] Ryan S, Frise,Fangwen Rao and Daniel TO, Connor, (2009). Matrix Metalloproteinases: Discrete Elevations in Essential Hypertension and Hypertensive End-Stage Renal Disease. Clinical and experimental hypertension, 31 (7): 521- 533.

[37] Berg G, Miksztowicz V, Schreier L. (2011). Metalloproteinases in metabolic syndrome. Clinica Chimica Acta, $412: 1731$ - 1739 .

[38] Tomohiro Kurihara, Ryoko Shimizu-Hirota, MD, Masayuki Shimoda, Takeshi Adachi, Hideyuki Shimizu, Stephen J.
Weiss, Hiroshi Itoh, Shingo Hori, Naoki Aikawa, Yasunori Okada, (2012). Neutrophil-Derived Matrix Metalloproteinase 9 Triggers Acute Aortic Dissection. Circulation, 126: 30703080 .

[39] Li YY, McTiernan CF, Feldman AM. (2000). Interplay of matrix metalloproteinases, tissue inhibitors of metalloproteinases and their regulators in cardiac matrix remodeling. Cardiovasc Res, 46: 214- 224.

[40] Zervoudaki A, Economou E, Pitsavos C, Vasiliadou K, Aggeli C, Tsioufis K, Toutouza M, Stefanadis C, Toutouzas P. (2004). The effect of $\mathrm{Ca}^{2+}$ channel antagonists on plasma concentrations of matrix metalloproteinase-2 and -9 in essential hypertension. Am J Hypertens, 7: 273-276.

[41] Ahmed SH, Clark LL, Pennington WR, Webb CS, Bonnema DD, Leonardi AH, McClure CD, Spinale FG, Zile MR. (2006). Matrix metalloproteinases/tissue inhibitors of metalloproteinases: Relationship between changes in proteolytic determinants of matrix composition and structural, functional, and clinical manifestations of hypertensive heart disease. Circulation, 113:2089- 2096.

[42] Josep Redón, Maria R, Oliva, Carmen Tormos, Vicente Giner, Javier Chaves, Antonio Iradi, Guillermo T. Sáez, (2003). Antioxidant Activities and Oxidative Stress Byproducts in Human Hypertension. Hypertension, 41: 1096- 1001.

[43] Espinosa O, Jimenez-Almazan J, Chaves F J, Tormos M C, Clapes S, Iradi A, Salvador A, Fandos M, Redón J, Sáez G T.(2007). Urinary 8-oxo-7,8-dihydro-2'-deoxyguanosine(8oxo-dG), a reliable oxidative stress marker in hypertension. Free Radic. Res, 41:546- 554.

[44] Ferri, J, Martinez-Hervas S, Espinosa O, Fandos M, Pedro T, Real JT, Chaves F J, Cerdá C, Sáez G, Ascaso J F. (2008). 8oxo-7,8-dihydro-2'-deoxyguanosine as a marker of DNA oxidative stress in individuals with combined familiar hyperlipidemia . Med. Clin. (Barc.), 131:1-4.

[45] Maria L, Mansego, Josep Redon, Sergio Martinez-Hervas,Jose $\mathrm{T}$ Real, Fernando Martinez, Sebastian Blesa,Veronica Gonzalez-Albert, Guillermo T Saez, Rafael Carmena, Felipe J, Chaves, (2011). Different Impacts of Cardiovascular Risk Factors on Oxidative Stress. Int. J. Mol. Sci, 12: 6146- 6163.

[46] Subash P, Gurumurthy P, Sarasabharathi A, Cherian K M. (2010). Urinary 8-OHdG: A Marker of Oxidative Stress to DNA and Total Antioxidant Status in Essential Hypertension with South Indian Population. Indian Journal of Clinical Biochemistry, 25 (2):127- 132.

[47] Mussalo H, Vanninen E, Hartikainen J. (2003). NT-proANP and BNP in renovascular and in severe and mild essentila hypertension. Kidney Blood Press R, 26:34-41.

[48] Pfister R, Tan D, Thekkanal J, Erdmann E, Schneider CA. (2008). Predictive value of NT-pro-BNP for the noncardiologist. A study on 573 hospitalized patients with cardiovascular disease. Dtsch Med Wochenschr, 133 (12): 564 -569 .

[49] Schöneich C. (1999). Reactive oxygen species and biological aging: a mechanistic approach. Exp Gerontol, 34: 19-34.

[50] Das SK, Sanyal K, Basu A. (2005). Study of urban community survey in India: Growing trend of high prevalence in developing country. Int J Med Sci, 2:70- 78. 
[51] Busserolles J, Mazur A, Gueux E, Rock E, Rayssiguier Y.(2002). Metabolic syndrome in the rat: Females are protected against the pro-oxidant effect of a high sucrose diet. Exp Biol Med, 227: 837- 842.

[52] Torres-Ramalho, Paulo; Araújo, José Paulo; Bettencourt, Paulo; Moura, Luís M. (2012). Natriuretic peptides in aortic stenosis.Rev Port Cardiol, 31:655- 660.
[53] Wang TJ, Larson MG, Levy D, Leip EP, Benjamin EJ, Wilson PW, Sutherland P, Omland T, Vasan RS. (2002). Impact of age and sex on plasma natriuretic peptide levels in healthy adults. Am J Cardiol, 90: 254 - 558. 